\title{
Role of Smoking in Male Infertility
}

\author{
Subrat Panda*, Ananya Das, Anusuya Sharma and Vinayak Jante \\ Department of Obstetric Gynecology, India
}

ISSN: 2640-9666

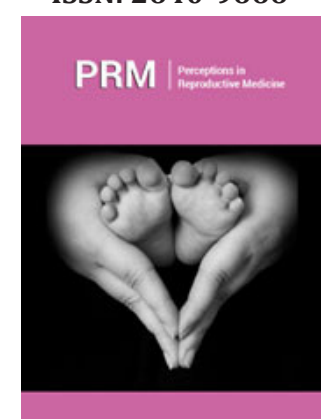

*Corresponding author: Subrat Panda, Department of Obstetric Gynecology, India

Submission: 海 October 04, 2021

Published: 望 October 21, 2021

Volume 4 - Issue 5

How to cite this article: Subrat Panda ,Ananya Das, Anusuya Sharma, Vinayak Jante. Role of Smoking in Male Infertility. Perceptions Reprod Med. 4(5). PRM 000597. 2021.

DOI: 10.31031/PRM.2021.04.000597

Copyright@ Subrat Panda, This article is distributed under the terms of the Creative Commons Attribution 4.0 International License, which permits unrestricted use and redistribution provided that the original author and source are credited.

\begin{abstract}
Objectives: Cigarette smoking is associated with subfertility in males and may lead to a decrease in sperm concentration, lower sperm motility, and a reduced percentage of morphologically normal sperms. The objective of the study is to find out the effect of smoking with semen parameters and the relationship between amount of cigarette smoking and semen parameters.
\end{abstract}

Method: This is a hospital-based cohort study conducted in the Infertility clinics of NEIGRIHMS from December 2019 for a period of 1year. 254 male partners were enrolled for the study. Routine semen analysis was carried out under light microscopy according to WHO guidelines.2010. Data was analysed to find out relation of smoking with semen parameters and the relationship between amount of cigarette smoking per day and semen parameters by using SPSS Software Version 21.

Result: In the one-year period we had 140 cases of non-smoker and 114 cases of smokers. On analysis of the findings of sperm count, smokers have relative risk of 2.45 of getting oligozoospermia ( $p$ value $=0.0005$ ). Smokers have relative risk of 1.5 of having total motility $<50 \%$. For progressive motility, smokers have relative risk of 2.01 of getting progressive motility<32\%. No dose relationship with abnormal semen parameter.

Conclusion: Smoking affected sperm count, and total and progressive motility but there was no dose and effect relationship between number of cigarettes per day and its affect on semen parameter.

Keywords: Smoking; Non-smokers; Total motility; Progressive motility; Vitality; Morphology

\section{Introduction}

Male infertility plays a key role in conception difficulties of up to $40-50 \%$ infertile couples [1]. Although in some men, a specific disorder may be present; however, no apparent reason for infertility can be found. This has drawn attention to the impact of lifestyle and environmental factors, especially diet, obesity, smoking, alcohol intake, recreational drug use, and exposure to environmental toxins, on reproductive health of such men [2]. Many studies have shown that cigarette smoking has a deleterious effect on both male and female reproductive systems and fertility [3]. Nowadays, the population of cigarette smokers is increasing despite the facts about its effects and hazards to human health and this number is reaching one third of worldwide population in the 15 years and above age group. Cigarette smoke contains a large number of substances which are recognized as carcinogens and mutagens including radioactive polonium, cadmium, benzopyrene, dimethylbenzanthracene, dimethyl nitrosamine, naphthalene and methnaphthalene. However, the toxicity of many of these constituents in cigarette smoke has not been well assessed for their effect on the function of human spermatozoa.

Although the effect of cigarette smoking on sperm function was noticed a long time ago, the mechanism by which tobacco smoke affects sperms remains poorly understood [4]. 
Thus, the impact of cigarette smoking on male fertility and sperm characteristics remains a highly controversial issue. Around 15 number of cigarette smoking may be associated with subfertility in males and may lead to a decrease in sperm concentration, lower sperm motility, and a reduced percentage of morphologically normal sperms [5,6]. Many studies on the effect of smoking on semen quality have been carried out worldwide $[6,7]$. Some of these studies showed that cigarette smoking results in a reduction of sperm concentration, motility and normal morphology [6]. Others have shown that cigarette smoking has a detrimental effect on sperm quality [8]. There were many other studies with inconsistent and conflicting results.

\section{Materials and Methods}

This is a hospital-based cohort study conducted in the Infertility clinic of NEIGRIHMS from December 2019 for a period of 1year.

\section{Inclusion criteria}

All males having abnormal semen parameters specially, counts, motility, morphology and vitality.

\section{Exclusion criteria}

a. Males with abnormal genital examination (varicocele, undescended testicles, hydrocele, small sized testes);

b. Males with azoospermia

c. Males with history of mumps.

d. Males with history of surgery for inguinal hernia, orchidopexy, or any scrotal surgery.

e. Males with chronic medical illness (diabetes mellitus, hypertension, thyroid disease, cancer patients, and tuberculosis; and

f. Males who had quit smoking for a period of less than 6 months. Seminal fluid was collected via masturbation into sterile wide mouth plastic container after 3-5 days of sexual abstinence in a room near the laboratory.
Samples with partial spillage were rejected; one sample per male was included in the study. Samples were allowed to liquefy at $37{ }^{\circ} \mathrm{C}$ environment, followed by analysis at room temperature. Routine semen analysis was carried out under light microscopy according to WHO guidelines 2010. Semen analysis comprised of ejaculate volume, $\mathrm{pH}$, viscosity, and sperm concentration (million/ $\mathrm{ml}$ ), which were determined using Makler Chamber. Sperm motility was determined as percentage of motile spermatozoa, and morphology as percentage of normally-shaped spermatozoa. The volume of ejaculate was measured by aspirating the whole sample into a graduated pipette, the total sperm count was measured by multiplying the sperm concentration by the volume of ejaculate. For sperm morphology, slides were smeared with a small volume of semen, and allowed to dry at room air temperature, stained with eosin, then read for normal or abnormal sperms according to WHO criteria 2010. Data was analysed to find out relation of smoking with semen parameters and the relationship between amount of cigarette smoking and semen parameters by using SPSS Software Version 21.

\section{Result \& Observations}

In the one-year period we had 140 cases of non-smokers and 114 cases of smokers. When the semen parameters in terms of sperm count was analyzed, smokers have relative risk of 2.45 getting oligozoospermia ( $p$ value $=0.0005$ ). In case of total motility $<50 \%$, smokers have relative risk of 1.5 ( $p$ value 0.015 ). For progressive motility, smokers have relative risk of 2.01 of getting progressive motility $<32 \%$ ( $p$ value $<0.0001$ ). But for vitality and morphology, we did not get any significant difference between smokers and non-smokers. In our study, the participants in smoking group were taking cigarettes varying from 5 to $>20$ cigarettes per day. We also analysed to see any correlation of number of cigarettes and relationship to the semen parameters by applying Pearsons Correlation test $\mathrm{R}^{2}$ (Count $=0.007$, Total Motility $=0.014$, Progressive motility $=0$, Morphology $=0.012$, Vitality $=0.098$ ) but we did not get any significant correlation between number of cigarettes and semen parameters as shown in the graphical representation (Table 1); (Figure $1 \& 2$ ).

Table 1: Effect of smoking on semen parameters.

\begin{tabular}{|c|c|c|c|c|c|}
\hline \multicolumn{2}{|c|}{ Semen Parameters } & Smoker & Non-Smoker & Relative Risk & P Value \\
\hline \multirow{2}{*}{ Count } & Normal count & 78 & 122 & \multirow{2}{*}{2.45} & \multirow{2}{*}{0.0005} \\
\hline & oligospermia & 36 & 18 & & \\
\hline \multirow{4}{*}{ Motility } & Normal motility & 44 & 73 & \multirow{2}{*}{1.5} & \multirow{2}{*}{0.015} \\
\hline & Motility $<50 \%$ & $71 \mathrm{a}$ & 66 & & \\
\hline & $\begin{array}{l}\text { Progressive Motility } \\
\qquad(<32 \%)\end{array}$ & 87 & 53 & \multirow{2}{*}{2.01} & \multirow{2}{*}{$<0.0001$} \\
\hline & $\begin{array}{l}\text { Non-Progressive } \\
\text { Motility }(>32 \%)\end{array}$ & 27 & 87 & & \\
\hline \multirow{2}{*}{ Vitality } & Vitality $(<58 \%)$ & 29 & 38 & \multirow{2}{*}{0.93} & \multirow{2}{*}{0.759} \\
\hline & Vitality $(>58 \%)$ & 85 & 102 & & \\
\hline \multirow{2}{*}{ Morphology } & Morphology $(<4 \%)$ & 50 & 54 & \multirow{2}{*}{1.13} & \multirow{2}{*}{0.3928} \\
\hline & Morphology $(<4 \%)$ & 64 & 86 & & \\
\hline
\end{tabular}



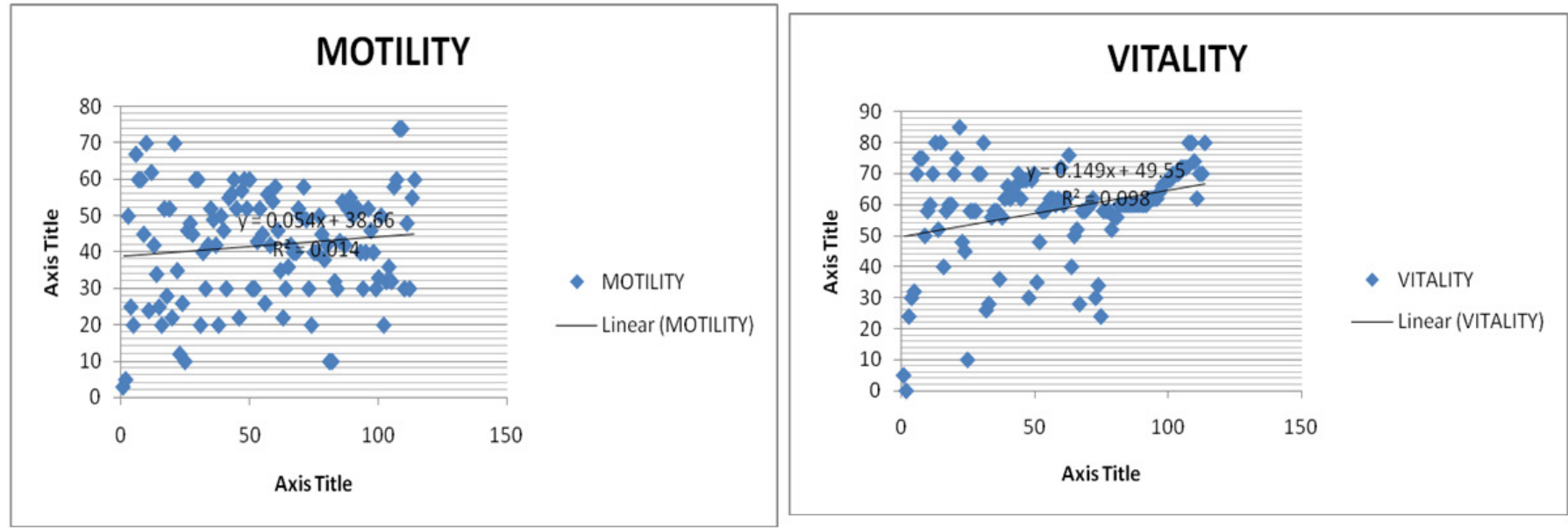

Figure 1: Correlation of sperm motility and vitality with smoking.
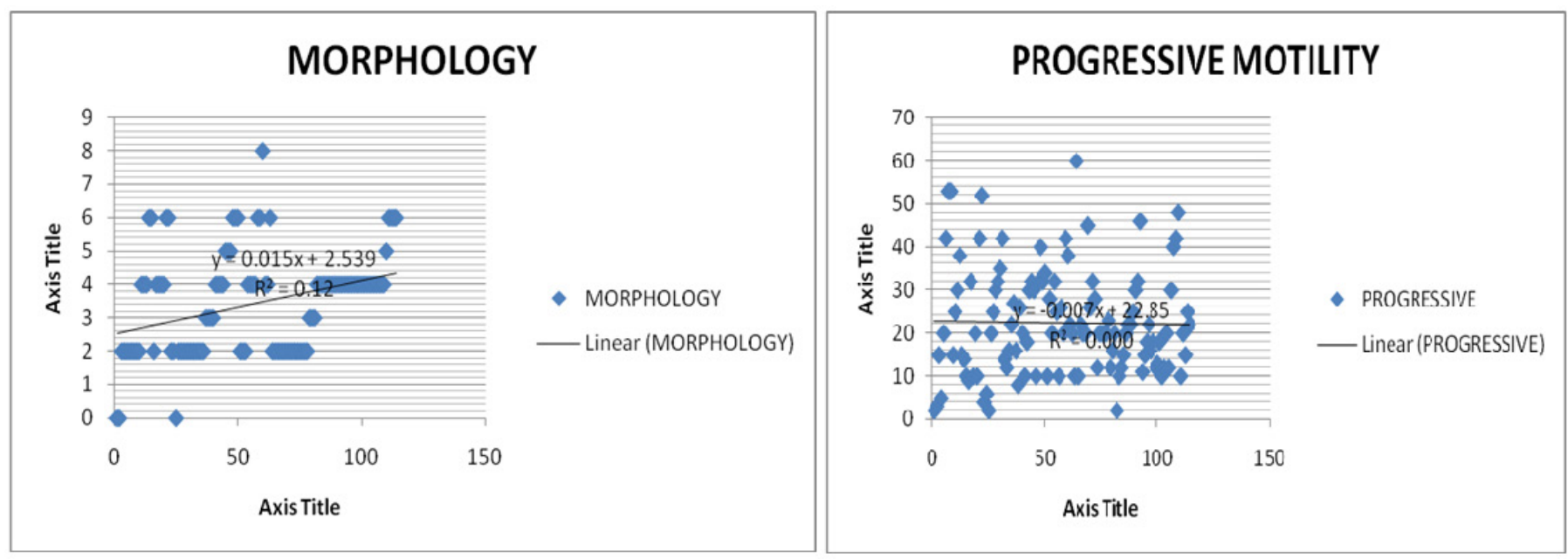

Figure 2: Correlation of sperm morphology and progressive motility with smoking.

\section{Discussion}

Despite worldwide anti-smoking campaigns, cigarette smoking is very common. In India, the prevalence of smoking in males in reproductive age group (15-69 years) is $24 \%$. In our study, we had $44.8 \%$ smoker males attending our infertility clinic. The exact mechanism of action of nicotine on semen parameters is not known. Smokers have lower seminal zinc levels than non-smokers, with associated decrease in sperm concentration, motility, and morphology. Interestingly, smokers with normal seminal zinc concentrations did not experience the same degree of abnormal semen parameters as those smokers with decreased seminal zinc, suggesting that zinc concentrations could play a role [7].

In our study, smokers have a relative risk of 2.45 of having oligozoospermia than the non-smokers. Smokers have a relative risk of 2.01 of decreased progressive motility $(<32 \%)$ than the non-smokers. But we did not get any significant difference in morphology and vitality. We also did not get any correlation between the number of cigarettes/day and semen parameters. A cross-sectional analysis of 2542 healthy men from 1987 to 2004 by Ramlau-Hansen [6] found that on semen analysis, cigarette smokers had lower semen volumes, sperm counts, and percentage of motile sperm compared to men who did not smoke. In another large cohort of 1786 men undergoing infertility workup (655 smokers and 1131 non-smokers), Kunzle et al. [8] demonstrated that smoking was associated with decrease in sperm density (15.3\%), total sperm counts (17.5\%), and total motile sperm (16.6\%) compared with non-smokers. Zhang et al. [9] studied 362 Chinese men attending an infertility clinic and found that smokers demonstrated decreased semen volumes, sperm concentrations, and rates of forward progression compared with non-smokers.

Merino and colleagues, [10,11] who studied 358 Mexican men stratified into 3 categories based on the number of cigarettes smoked per day, also confirmed this type of dose dependency. The authors confirmed the effects of smoking on reduced sperm density and abnormal morphology, but also extended these findings to note that men who smoked $<10$ cigarettes per day experienced significant changes in their semen analysis parameters. Therefore, even "light" smokers appeared to be at risk for adverse effects on fertility. Mahadeo Maroti et al in their study showed There was no 
significant difference between mild, moderate and heavy smoker. Reecha Sharma et al. [2] found deterioration of semen quality is more pronounced in moderate and heavy smokers. Künzle et al. [8] prospectively studied 2,105 participants, of whom 839 persons (39.9\%) were smokers and 1,266 persons (60.1\%) were nonsmokers [12-14]. Sperm concentration, motility, and morphology were significantly affected in smokers. Nevertheless, sperm vitality, ejaculate volume, and progressive motility concentrations were slightly but not significantly reduced among smokers. In our study, we definitely found that smoking affected sperm count and motility but no dose dependent relationship between smoking and semen parameters could be elicited.

\section{Conclusion}

Smoking definitely affects male fertility adversely. Healthcare providers should facilitate individuals to quit smoking by educating, monitoring and constant support. The data on smoking and male fertility reinforce the preferred preventive approach of discouraging smoking and eliminating exposure to tobacco smoke among both males and females in general, and males in particular, while trying to conceive.

\section{References}

1. Kumar N, Singh AK (2015) Trends of male factor infertility, an important cause of infertility: A review of literature. J Hum Reprod Sci 8(4): 191196.

2. Sharma R, Kelly RB, Jennifer M Fedor, Agarwal A (2013) Lifestyle factors and reproductive health: Taking control of your fertility. Reprod Biol Endocrinol 11: 66.

3. Zenzes MT (2000) Smoking and reproduction: gene damage to human gametes and embryos. Hum Reprod Update 6(2): 122-131.
4. Windham GC, Swan SH, Fenster L (1992) Parental cigarette smoking and the risk of spontaneous abortion. Am J Epidemiol 135(12): 1394-1403.

5. Lewin A, Gonen O, Orvieto R, Schenker JG (1991) Effect of smoking on concentration, motility and zona-free hamster test on human sperm. Arch Androl 27(1): 51-54.

6. Zinaman MJ, Brown CC, Selevan SG, Clegg ED (2000) Semen quality and human fertility: A prospective study with healthy couples. J Androl 21(1): 145-153.

7. Liu RZ, Gao JC, Zhang HG, Wang RX, Zhang ZH, et al. (2010) Seminal plasma zinc level may be associated with the effect of cigarette smoking on sperm parameters. J Int Med Res 38(3): 923-928.

8. Künzle R, Mueller MD, Hänggi W, Birkhäuser $M H$, Drescher $H$, et al. (2003) Semen quality of male smokers and non-smokers in infertile couples. Fertil Steril 79(2): 287-291.

9. Zhang JP, Meng QY, Wang Q, Zhang LJ, Mao YL, et al. (2000) Effect of smoking on semen quality of infertile men in Shandong, China. Asian J Androl 2(2): 143-146

10. Olmedo BS, Chillik C, Kopelman S (2001) Definition and causes of infertility. Reprod Biomed Online 2(1): 41-53.

11. Augood C, Duckitt K, Templeton AA (1998) Smoking and female infertility: A systematic review and meta-analysis. Hum Reprod 13(6): 1532-1539.

12. Osser S, Liedholm P, Ranstam J (1984) Depressed semen quality: A study over two decades. Arch Androl 12(1): 113-116.

13. Arabi M, Moshtaghi $H$ (2005) Influence of cigarette smoking on spermatozoa via seminal plasma. Andrologia 37(4): 119-124.

14. Chia SE, Lim ST, Tay SK, Swee TL (2005) Factors associated with male infertility: A case-control study of 218 infertile and 240 fertile men. Brit J Obstet Gynaecol 107: 55-61.

For possible submissions Click below: 